\title{
XMM-NEWTON AND OPTICAL OBSERVATIONS OF WZ SAGITTAE IN QUIESCENCE
}

\author{
K. Mukai ${ }^{1,2}$ and J. Patterson ${ }^{3}$
}

WZ Sge is the prototype of a subclass of dwarf novae with an extremely long recurrence period. In addition, it exhibits rapid oscillations at $27.87 \mathrm{~s}$ and $28.96 \mathrm{~s}$. We present our preliminary analysis of XMM-Newton and optical observations taken in 2003 May, almost 2 years after the 2001 outburst.

We observed WZ Sge with XMM-Newton for $\sim 10$ ksec on 2003 May 16. WZ Sge was detected at a combined total of $\sim 5 \mathrm{cts} \mathrm{s}^{-1}$ in the three EPIC cameras. Our spectral analysis reveals a multi-temperature plasma, with a flux of $7.0 \times 10^{-12} \mathrm{ergs} \mathrm{cm}^{-2} \mathrm{~s}^{-1}$ in the $2-10 \mathrm{keV}$ band, much brighter than the $2.9 \times 10^{-12}$ ergs cm${ }^{-2} \mathrm{~s}^{-1}$ measured from the 1996 May $A S C A$ data. Inferred $0.2-10 \mathrm{keV}$ luminosity of WZ Sge was $2.5 \times 10^{30} \mathrm{ergs} \mathrm{s}^{-1}$ for the adopted distance of $43 \mathrm{pc}$ (Thorstensen 2003).

We also obtained optical photometry of WZ Sge with the MDM $2.4 \mathrm{~m}$ telescope on 2003 May 15-20. In these data, we detect a coherent periodicity of $28.9593(1) \mathrm{s}$, with an amplitude of $\sim 1.2 \%$. There is some power at this period in the $2-10 \mathrm{keV}$ band $\mathrm{X}$-ray light curve, although we cannot claim an independent detection (Figure 1). We do not detect the $27.87 \mathrm{~s}$ signal either in the optical or in the X-rays.

Patterson et al. (1998) favored the magnetic accretor model as the origin of the rapid oscillations in WZ Sge, based on a weak detection of the $27.87 \mathrm{~s}$ in the 1996 May ASCA observation. In this model, we expect the spin period pulse to be persistent; and, if it ceases, we would not expect other high frequency signals to be able to persist. Thus, our results present a serious challenge to this interpretation. If WZ Sge is magnetic, it does not resemble any other magnetic binaries we know about.

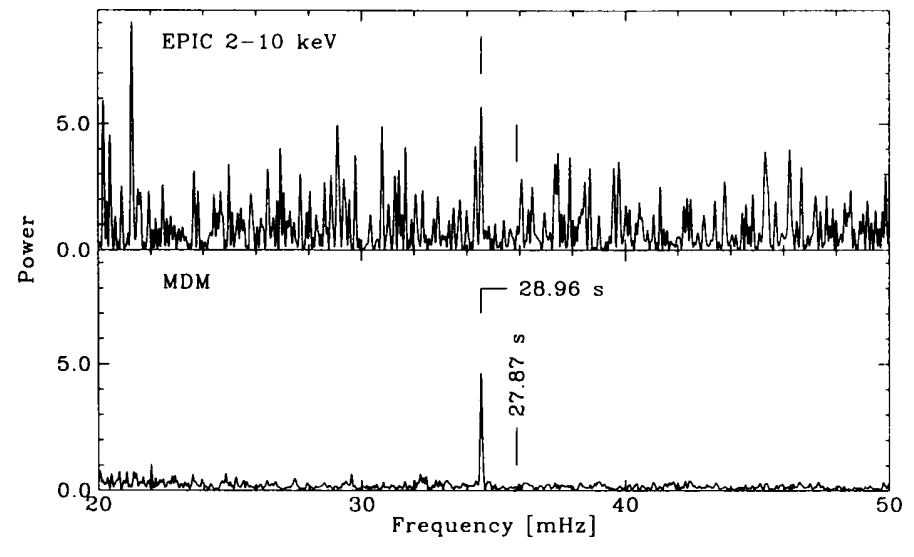

Fig. 1. Power spectra of WZ Sge from XMM-Newton and optical data both taken in 2003 May:

Alternatively, the rapid oscillations in WZ Sge may be due to non-radial $g$-mode pulsation of the primary. In addition to the difficulties enumerated by Welsh et al. (2003), this model needs a mechanism to generate X-ray modulation at the dominant period du jour, albeit weakly.

It appears that, after 25 years of observations, with some of the most powerful instruments on and above Earth, we are no closer to understanding WZ Sge!

\section{REFERENCES}

Patterson, J., Richman, H., Kemp, J. \& Mukai, K. 1998, PASP 110,403

Thorstensen, J.R. 2003, AJ 126, 3017

Welsh, W.F., Sion, E.M., Godon, P., Gänsicke, B.T., Knigge, C., Long, K.S. \& Szkody, P. 2003, ApJ 599, 509

\footnotetext{
${ }^{1}$ Code 662, NASA/Goddard Space Flight Center, Greenbelt, MD 20771, USA (mukai@milkyway.gsfc.nasa.gov).

${ }^{2}$ Also Universities Space Research Association.

${ }^{3}$ Department of Astronomy, Columbia University, 550 West 120th Street, New York, NY 10027, USA (jop@astro. columbia.edu).
} 